\title{
Hypernatural Monitoring: A Social Rehearsal Account of Smartphone Addiction
}

\author{
Samuel P. L. Veissière ${ }^{1,2,3,4 *}$ and Moriah Stende $\left.\right|^{1,3,4}$ \\ ${ }^{1}$ Department of Psychiatry, McGill University, Montreal, QC, Canada, ${ }^{2}$ Department of Anthropology, McGill University, \\ Montreal, QC, Canada, ${ }^{3}$ Raz Lab in Cognitive Neuroscience, McGill University, Montreal, QC, Canada, ${ }^{4}$ Culture, Mind, and \\ Brain Program, McGill University, Montreal, QC, Canada
}

We present a deflationary account of smartphone addiction by situating this purportedly antisocial phenomenon within the fundamentally social dispositions of our species. While we agree with contemporary critics that the hyper-connectedness and unpredictable rewards of mobile technology can modulate negative affect, we propose to place the locus of addiction on an evolutionarily older mechanism: the human need to monitor and be monitored by others. Drawing from key findings in evolutionary anthropology and the cognitive science of religion, we articulate a hypernatural monitoring model of

OPEN ACCESS

Edited by: Maurizio Tirassa,

Università degli Studi di Torino, Italy

Reviewed by:

Giulia Piredda,

Istituto Universitario di Studi Superiori

di Pavia (IUSS), Italy

Yasmina Jraissati,

American University of Beirut,

Lebanon

${ }^{*}$ Correspondence:

Samuel P. L. Veissière samuel.veissiere@mcgill.ca

Specialty section: This article was submitted to Theoretical and Philosophical

Psychology,

a section of the journal

Frontiers in Psychology

Received: 16 November 2017 Accepted: 29 January 2018 Published: 20 February 2018

Citation:

Veissière SPL and Stendel M (2018) Hypernatural Monitoring: A Social Rehearsal Account of Smartphone Addiction. Front. Psychol. 9:141. doi: 10.3389/fpsyg.2018.00141 smartphone addiction grounded in a general social rehearsal theory of human cognition. Building on recent predictive-processing views of perception and addiction in cognitive neuroscience, we describe the role of social reward anticipation and prediction errors in mediating dysfunctional smartphone use. We conclude with insights from contemplative philosophies and harm-reduction models on finding the right rituals for honoring social connections and setting intentional protocols for the consumption of social information.

Keywords: smartphone addiction, social neuroscience, evolutionary anthropology, predictive-processing, cultural affordances, social rehearsal, hungry ghosts

\section{INTRODUCTION}

As this paper was undergoing final review, a new wave of editorials about the noxious effects of smartphone use was sweeping the news. Major Apple shareholders, backed by petitions from customers, were now demanding that the tech giant address the growing problem of smartphone addiction and its impact on children's development (Kawa, 2018). As cognitive scientists who have studied the impact of the internet on human behavior (Veissière, 2016a,b), our aim is to present a nuanced view of the relationship between mobile information technology and human well-being. While we agree that excessive smartphone use can be detrimental to mental health, we aim to recast current understandings of the mechanisms involved in these addictive patterns in a broader evolutionary focus.

In this paper, we offer the provocative claim that current moral panics over smartphone addiction overlook a factor of fundamental importance: there is nothing inherently addictive about mobile technology. We suggest, rather, that it is the social expectations and rewards of connecting with other people and seeking to learn from others that induce and sustain addictive relationships with smartphones. Much has been said about Internet addiction and the new medias and technologies that connect us and make us lonely at the same time, leading to adverse mental health consequences (Twenge, 2017).The deeply prosocial 
nature of these mechanisms, however, is often understated. Compulsive smartphone use, we claim, is not so much antisocial as fundamentally social. Specifically, we argue that mobile technology addiction is driven by the human urge to connect with people, and the related necessity to be seen, heard, thought about, guided, and monitored by others, that reaches deep in our social brains and far in our evolutionary past.

Smartphones, we claim, provide a potentially unhealthy platform for another healthy impulse. As we will see, they can also enable us to remember and celebrate the role of other people in making us who we are, and help us treasure the bonds that make us a uniquely social species.

In fleshing out the social roots of smartphone addiction and by extension, of human behavior and well-being - we do not intend to produce a general meta-theory that dismisses other, non-social forms of excessive smartphone use. The hypersociality of smart-device addiction, rather, may likely occur on a continuum from the directly social to the indirectly social.

Playing video-games, outsourcing difficult tasks like memorizing schedules or spatial orientation, and having instant access to news and information are among of battery of everyday smartphone functions that are known to be highly addictive (Alter, 2017). At a glance, these domains are not readily apparent as social. From an evolutionary perspective, however, the human capacity to function optimally in any environment (and indeed human intelligence itself) is predicated on having access to a large, cumulative repertoire of contextually relevant cultural information devised by others, and that no single individual could invent on her own, or recreate alone in her own lifetime (Henrich, 2016; Mercier and Sperber, 2017). Seeking news and information, to put it simply, are ways to learn from others, and to stay updated on culturally relevant events and people. Video-gaming is similarly underpinned by social dimensions that may not be readily visible to users and critics alike. While many video-games involve explicit social rewards from playing online with other users (Snodgrass et al., 2016) other uniquely addictive smartphone games like Candy crush do not. The unpredictable rewards derived from so-called "ludic loops" of increased difficulty (Alter, 2017), as we expand in the Section "Predictive-Processing and Smartphones," typically activate neurobiological systems that increase reward-seeking behavior and addictions in other domains (West et al., 2015). In the next section, we present findings supporting the hypothesis that most smartphone notifications, from email and texting to social media, modulate addictive behavior through the anticipation of social rewards. The rewards derived from playing games, however, are social in more indirect ways. The human drive for gaming and competition, indeed, is also rooted in social evolutionary mechanisms, in which intra- and inter-group competition have helped drive the iterative spread of skill, knowledge, and technology from generation to generation (Bell et al., 2009; Richerson et al., 2016). In seeking to excel at a difficult game, we are rehearsing excellence in particular domains of skill, but also in the domain of social competition itself. Smartphones, as we will argue, provide a hyper-efficient extension of deep evolutionary urges for connection with others, learning from others, but also comparing ourselves to and competing with others.

\section{The Sociality of Smartphone Use}

When it comes to smartphone use, current scientific literature and intuitive wisdom are overwhelmingly pessimistic, warning us of the dangers these new technologies enable. According to current research, smartphone use is associated with depression (Steers et al., 2014; Andreassen et al., 2016), materialism (Lee et al., 2014; Twenge, 2017), and social anxiety (Billieux et al., 2015; Emanuel et al., 2015; Hussain et al., 2017), spawning a generation of anti-social, chronically anxious, self-obsessed 'zombies' (Lu and Lo, 2017). While these findings raise important concerns about the 'dark side' of smartphone use, they tend to focus on new technologies as the sole locus of addiction and pathology. We propose to bring this problem into a broader evolutionary focus, and will go on to argue that the current 'smartphone obsession' is neither grounded in, nor indicative of a paradigmatic shift in the psychosocial context in which human experience is invariably framed. Popular accounts, we argue, miss the mark on a crucially important factor: it is not so much smartphones themselves that are addictive, but rather the sociality that they afford. We insist that this drive for sociality is a fundamental feature of human evolution that predates smartphones by hundreds of thousands - by some accounts several millions - of years (Hrdy, 2007). Simply put, smartphone addiction is hyper-social, not anti-social.

There is ample evidence to support the claim that smartphone use is inherently prosocial, and by extension, that this prosociality is a core locus of smartphone addiction. First, the majority of smartphone use is spent on social activities such as social networking, text messaging, and phone calls (Li and Chung, 2006; Lopez-Fernandez et al., 2014). Even less interactive smartphone use, like information seeking or surfing the web, has now become implicitly social: 'likes', views, and comments are social indices of prestige and collective attention. Second, individuals who use their devices for primarily social purposes are quicker to develop habitual smartphone use (Van Deursen et al., 2015). These findings suggest that it is not just the smartphone itself that is addictive but rather the-direct or indirect-social interaction it enables.

Gendered dimensions of smartphone addiction provide further clues into its inherent sociality. Current findings in evolutionary psychology and social neuroscience indicate that women are on average more proficient at social cognition and tend to display more prosocial behavior than men (Eckel and Grossman, 1998; Andreoni and Vesterlund, 2001; Meier, 2007; Laasch and Conaway, 2009; Rand et al., 2016; Soutschek et al., 2017; see Espinosa and Kováŕík, 2015 for alternate explanations). This gender discrepancy is maintained in smartphone use, with numerous studies showing that women use their phones for social purposes significantly more than men do (Tufekci, 2008; Van Deursen et al., 2015). According to our hypothesis, the prosocial nature of female smartphone use would render females more susceptible to addiction. Recent estimates confirm this view: females are more likely to develop addictive smartphone behaviors, experience more anxiety if they cannot use their smartphones, and feel less in control over checking their phones (Thompson and Lougheed, 2012; Van Deursen et al., 2015). 


\section{Imagined Other Minds Guide Our Expectations}

Despite minor gendered differences in social cognition, it is not controversial that humans as a whole are a prosocial species. Beyond amply documented findings in developmental psychology attesting to the intrinsic co-evolutionary links between cognition and sociality (Moll and Tomasello, 2007; Tomasello, 2009; Tomasello et al., 2012), recent research on mind-wandering has shown that a large part of our spontaneous mental lives is devoted to rehearsing social scenarios. A recent large-scale investigation using experience-sampling, for example, demonstrated that nearly half of waking time is spent in mindwandering episodes unrelated to the task at hand (Killingsworth and Gilbert, 2010). Although science on daydreaming often describes the consequences of a wandering mind (e.g., Mrazek et al., 2013), it is likely premature to believe that a cognitive function that occupies such a large percentage of mental life does not confer some adaptive benefit. To explain the ubiquity of mind-wandering, Poerio and Smallwood (2016) have proposed that the phenomenon is evolutionarily adaptive, serving as a platform for offline social cognition. Supporting this view, research shows that all but a small fraction of daydreaming involves social scenarios (Mar et al., 2012; Song and Wang, 2012). Moreover, mind-wandering and social cognition rely on shared neural activation, whereby the neural activity that occurs during daydreaming significantly overlaps with that of core social processes like mentalizing and perspective taking - the very processes that enable an individual to socially flourish (Poerio and Smallwood, 2016). Recent models on the evolution of depression help confirm this social hypothesis for the mechanisms of ordinary cognition. In a series of influential papers, Paul Andrews and colleagues have argued that 'depression' (a disorder characterized by cognitive rumination) confers specific social advantages to help keep social problems in mental focus. Again, it is of note that women (who are demonstrably more proficient than men at social cognition) experience depression at much higher rates than men. Andrews and colleagues see this as further evidence that a significant part of mental life is dedicated to rehearsing social scenarios (Andrews and Thomson, 2009; Andrews et al., 2012, 2015). All in all, a growing consensus between developmental psychology, cognitive neuroscience, and phenomenology strongly suggests that humans are almost always thinking about and through other people (Frith, 2002; Tomasello, 2009; Mar et al., 2012; Ramstead et al., 2016). The time is ripe, then, to elaborate a generalized social rehearsal theory of cognition. In the following sections, we expand on this theory and apply it to smartphone use.

\section{Social Media and Internet Notifications as Hyper-Natural Monitoring}

In a series of recent papers, Ramstead et al. (2016; see also Ramstead et al., 2017; Veissière, 2017) have described symbolically enriched human worlds as organized landscapes of "cultural affordances" grounded in mutual, recursively nested expectations about shared standards of behavior. 'Culture', on this view can be conceptualized as patterned allocations of attention; that is, the practice of selectively paying attention, ascribing meaning, and guiding behavior to certain features of the world according to what we expect others to also expect and pay attention to. While what is made salient through collectively shaped attentional preferences acquires different values and affords different experiences from group to group, the capacity for shared attention extrapolated to large groups of generalized 'like me' others is a species-wide disposition - the very disposition, mediated by joint-intentionality, that gives rise to cultural forms of life among Homo Sapiens (Ramstead et al., 2016; Veissière, 2017).

On this view, over the course of normal cognitive and social development, humans learn to see the world through the perspective of other people and intuitively imagine contextrelevant agents (usually imbued with prestige) to guide them in their actions (Veissière, 2017). From context to context and moment to moment, we outsource a large part of our thinking, feelings, and decision-making to sometimes explicit, most often implicit scenarios of the "what would so-and-so think, feel, or expect me to do" variety.

This reassuring feeling of being watched and guided by imaginary others has been hypothesized to play an important role in the evolution of cooperation, morality, organized religion, and large-scale social life (Whitehouse, 2004; Boyer, 2008; Norenzayan and Shariff, 2008; Atran and Henrich, 2010; Norenzayan et al., 2013). According to this view, often called the super-natural monitoring hypothesis, we fashioned our Gods and Spirits to better flesh out the imaginary agents that guide our ordinary cognition, consciousness, action, and moral attitudes.

Instant text messaging, email, and social media provide a platform for our hungry need to be connected, but also for our need to watch and monitor others, and better still, for our need to be seen, heard from, thought about, monitored, judged, and appraised by others. We might call this the hyper-natural monitoring hypothesis.

The prevailing - and hyperbolic - view on smartphone use is that it is a sly weapon, responsible for pandemic-like waves of mass loneliness, anxiety, insecurity, materialism, and narcissism among today's youth - particularly the so-called 'digital natives' born after 1994 (Roberts et al., 2015; Weiser, 2015; Pearson and Hussain, 2015; Twenge, 2017). As Jean Twenge has pointed out in her recent book on digital natives (Twenge, 2017), the advent of electronically mediated childhoods in the West was also concurrent with a general shift in parenting culture, and the rise of so-called 'helicopter parenting' in particular. Drawing on extensive survey research, she points out that children and youth born after 1994 spent considerably less unsupervised time socializing with their peers than their forebears, and significantly more time on electronic devices. While precise causality behind

\footnotetext{
1 "Helicopter parenting" is used as a derogatory term to describe obsessive parental supervision in most dimensions of children's lives. Although the phrase first appeared in the 1960's (Ginott, 1965/2009), it is often said to characterize the post-1980s childrearing culture of "hovering around" one's child. "Lawnmower parenting" (where one paves the way for children in all aspects of their lives), is sometimes used to describe more extreme forms of helicopter parenting. In November 2017, the Economist reported that parents in the United States and nine European countries (except for France), now spent 50\% more time with their children than in 1965 (The Economist, 2017).
} 
these two correlated factors cannot be ascertained, we can only note that youth who otherwise do not interact with their peers "in real life" (irl in internet lingo) seek to do so with the means available to their generation. Online-mediated life, more to the point, is always, already real life, and as such, it is inherently social.

What current moral panics about digital media often fail to consider, thus, is that the desire to see and be seen, and judge and be judged is precisely about other people. There is nothing abnormal, as such, about seeking self-worth through other people's point of view. We propose, thus, to think of this urge as fundamentally normal, and anchored in core mechanisms of social cognition that are distinct to our species. On our social rehearsal and monitoring view, smartphones simply equip us with a novel medium to channel innate human sociality. Their proclivity to induce addiction, in turn, simply points to how much others matter to us and how we want to matter to them.

\section{PREDICTIVE-PROCESSING AND SMARTPHONES}

If the primary motivation of smartphone use is prosocial, why can this technology lead to such negative outcomes? We turn to the science of addiction to describe how mobile technology in particular has sent us into a vortex of anxiety-inducing, hyperexcited, hyper-monitoring.

\section{A Brief Venture into the Neuroscience of Addiction}

The exact nature and neurochemical correlates of smartphone addiction are currently unknown (Elhai et al., 2017). Key insights from the neuroscience of learning and addiction, however, can offer important insights into our attachment to the strange flickering and buzzing bricks that seem to regulate our lives.

As we have seen, smartphone use is ance constitutive of and constituted by a complex landscape of sociality. This landscape, however, is also modulated by notifications from dozens of applications that deliver beeps and buzzes, mostly to alert us that another human has interacted with us. We should now consider where and how 'addiction' fits in this picture. Social interaction (digital or not) activates the dopaminergic reward circuits in the basal ganglia (See Krach et al., 2010 for a review). It is important to note that these same circuits are implicated in addictive drug use (Belin et al., 2009), compulsive video-gaming, and reward-seeking in general (West et al., 2015). These are circuits that are also responsible for associative learning: the process by which an individual learns to associate two stimuli (Hebb, 1976; Seger, 2006; Yin and Knowlton, 2006). For associative learning to occur, an initial exposure to a new stimulus must occur alongside a reflex-eliciting stimulus. With a smartphone, nearly all notifications that the user encounters elicit a social value and thus activate the dopaminergic reward circuit, leading the user to anticipate and seek these rewarding notifications. With each occurrence this link grows stronger, and the user will anticipate and seek these rewarding notifications, paving the road for habitual behavior.
The dopaminergic system regulates two functions that govern addiction: the anticipation of reward and outcome evaluation (Linnet, 2014). An important finding about dopamine and addiction, however, is that dopaminergic surges typically occur before the reward, or more precisely when a cue (e.g., a beep indicating that one can press a lever) signals the reliable delivery of a reward (e.g., from pulling a lever). Because arousal decreases with frequent and predictable exposure, reward anticipation is a much more powerful mediator of strong addictions than outcome evaluations of the stimulus itself (Fiorillo et al., 2003; van Holst et al., 2012). According to this finding, addictions become strongest when we cannot figure out the pattern of when to reliably expect them (van Holst et al., 2012). Behavioral scientists call these addiction-inducing patterns intermittent reinforcement or variable ratio schedules (Zuriff, 1970). Neuroscientists have identified that a cue triggering a behavior that yields a reward $50 \%$ of the time is by far the most anxiety-inducing of delivery schedules. A reward delivered 75\% of the time, for example can be reliably expected to deliver most of the time. A cue signaling a reward that delivers $25 \%$ of the time can similarly be expected not to deliver most of the time. Such high-predictability schedules (when the brain can reliably predict what is going to happen) typically trigger low arousal. At a $50 \%$ delivery rate, a reward schedule is still predictable enough to be enticing, but unpredictable enough to be anxiety-inducing (Fiorillo et al., 2003).

The point to take home here is that arousal is more highly correlated with reward anticipation than with the reward itself. When rewards become most unpredictable, in turn, arousal typically becomes negative, giving rise to anxiety (Figure 1).

Indeed, the beeps and buzzes of smartphone notifications provide just such an intermittent, variable, unpredictable, but uniquely desirable schedule of rarely met anticipation rewards, thus providing chaotic patterns of reward anticipation that trigger very strong modes of arousal. Because of the deeply social nature of the rewards our phones make us crave, we often become entrenched in vicious cycle of addiction (Figure 1).

\section{Cravings as Prediction Errors}

According to predictive-processing and free-energy theories of cognition, we do not immediately perceive the world as it is. Rather than directly respond to environmental stimulus, we first process information through our expectations. Immediate perception, in other words, first occurs through behavioral selfpredictions modulated by prior experience (Friston and Kiebel, 2009; Ramstead et al., 2016). On this view, our brains generate statistical models of the world based on prior learning to provide us with predictions of what will arise in experience and how to act accordingly. In doing so our brains predict upcoming sensory states and compare them with actual sensory states, minimizing the differences between these distributions through constant updates of priors and actions (i.e., learning) (Ramstead et al., 2016, 2017). As our perceptual system constantly attempts to reduce uncertainty by computing abysmal amounts of disordered information to make it predictable, discrepancies between prediction and perception - prediction errors in the lingo - become commonplace. Cravings, on this view, could 
Dopaminergic Activity in Response to Uncertain Stimuli

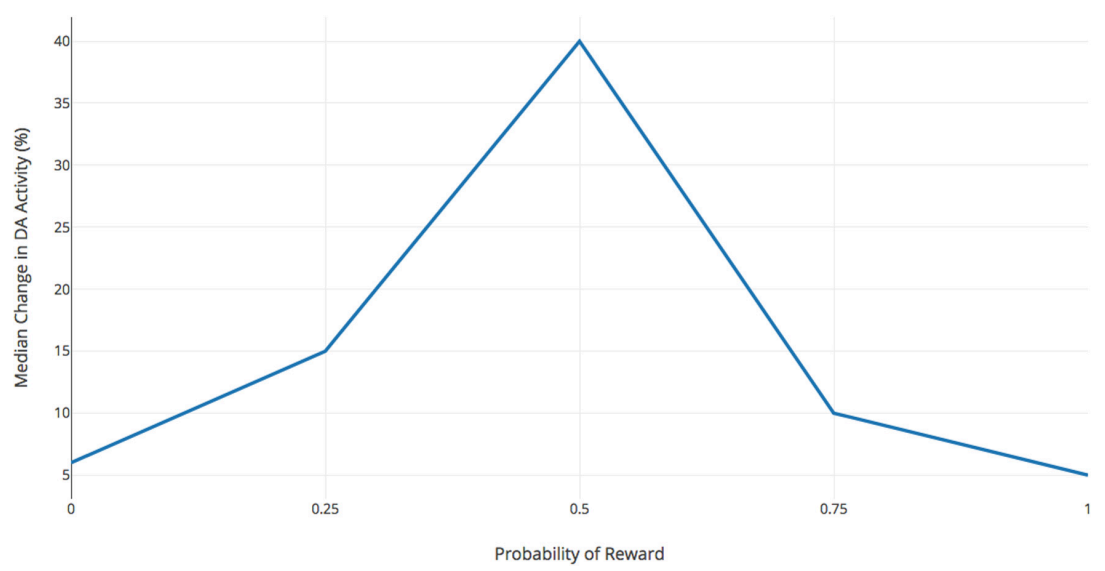

FIGURE 1 | Dopaminergic Activity in Response to Uncertain Stimuli (adapted from Fiorillo et al., 2003, Figure 3C). Average Sustained activation of dopamine neurons in a primate as a function of reward probability, whereby the greatest dopaminergic activity occurs when the reward is present half of the time.

A Unexpected Reward (Learning)

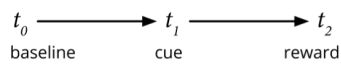

reward $\left[R_{\mathrm{r}}\right]$

state value $\left[\mathrm{V}\left(\mathrm{S}_{\mathrm{r}}\right)\right]$

error
$=R_{t}+V\left(S_{t}\right)-V\left(S_{t-1}\right)$

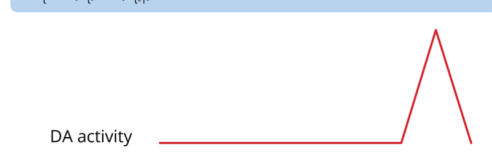

B Expected Reward

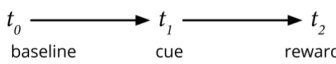

baseline cue reward

\begin{tabular}{ll|l|l} 
reward $\left[R_{t}\right]$ & 0 & 0 & 1 \\
\hline state value $\left[V\left(S_{t}\right)\right]$ & 0 & 1 & 0 \\
\hline
\end{tabular}

$\begin{array}{llll}\text { error } \\ =R_{t}+V\left(S_{t}\right)-V\left(S_{t}\right) & \mathbf{0} & \mathbf{1} & \mathbf{0}\end{array}$

DA activity
C Negative Prediction Error

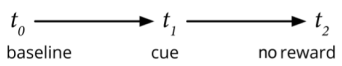

reward $\left[R_{\mathrm{t}}\right]$
state value $\left[\mathrm{V}\left(\mathrm{S}_{\mathrm{t}}\right)\right]$

\begin{tabular}{l|l|l}
0 & 0 & 0 \\
\hline 0 & 1 & 0
\end{tabular}

$\begin{array}{llll}\stackrel{\text { error }}{=} R_{t}+V\left(S_{t}\right)-V\left(S_{t-1}\right) & 0 & 1 & -1\end{array}$

DA activity

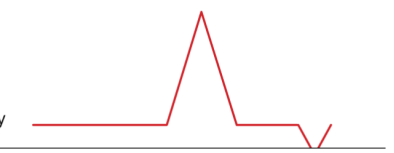

FIGURE 2 | Cue-activated reward anticipation and prediction errors and subsequent dopaminergic activity (adapted from Keiflin and Janak, 2015). (A) Before the cue is conditioned, the unexpected reward results in phasic activation of dopamine neurons and a positive reward prediction error. (B) Once a reward is conditioned, the cue (and not the reward) results in a positive reward anticipation and increased dopamine activity. (C) When the cue occurs but is met without the expected award, the result is a negative prediction error and a reduction of dopamine activity below baseline.

be conceptualized as prediction errors (Tobler et al., 2006) (Figures 2, 3).

As we mentioned above, associative learning and freeenergy models can explain the pervasive expectation that the anticipation of smartphone notifications predicts an upcoming social reward. In turn, the intermittent schedule of smartphone notifications promotes stronger anticipations and more compulsive expectations, subsequently inducing prediction errors and affective disappointment.

Notifications are cues for checking behavior that eventually becomes habitual, even without the initial alert (Oulasvirta et al., 2012; Elhai et al., 2017). Recent studies reveal the magnitude of this habitual checking behavior, with the average individual spending over $3 \mathrm{~h}$ a day on their smartphone (Alter, 2017), tapping, typing, or swiping an average of 2617 times every day (dscout, 2016). The majority of users go on to experience prediction errors in the form of hallucinations that their phone is vibrating, a phenomenon entitled phantom phone (Sauer et al., 2015). These prediction errors reinforce habitual phone checking behaviors, which are a common gateway to smartphone addiction (Oulasvirta et al., 2012). Prediction errors can also occur in more subtle, but equally frequent and distressing way when precise patterned expectations are not met: a beep that we hope may be a message from a loved one or a Instagram 'like', for example, may turn out to be an incoming spam email or a message from one's boss about an overdue task.

\section{THE DARK SIDE OF SOCIAL MONITORING?}

Key models of ordinary cognition, like predictive processing, free-energy, associative learning, and social rehearsal, all offer clues to elucidate the newfangled phenomenon of smartphone addiction. We have seen that smartphone addiction harnesses basic human proclivities for social monitoring and associative learning. While we largely intend this paper to add a hopeful note about potentially healthy social causes of smartphone addiction 


\section{A Unexpected Reward}

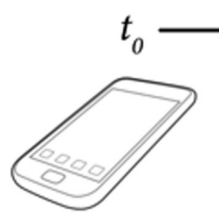

baseline

baseline

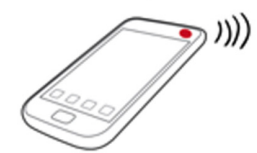

cue

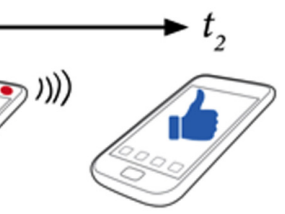

reward

unexpected facebook 'like'
B Expected Reward

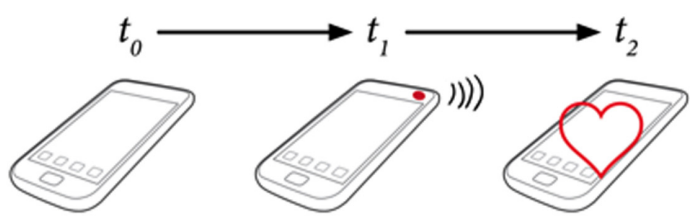

cue

reward

message from loved one

\section{baseline} notification

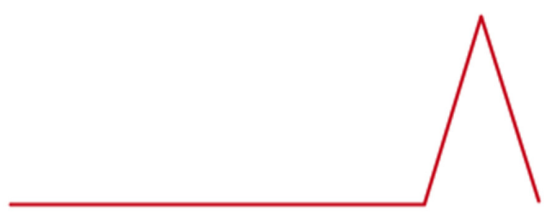

DA activity

\section{c Negative Prediction Error Type 1}
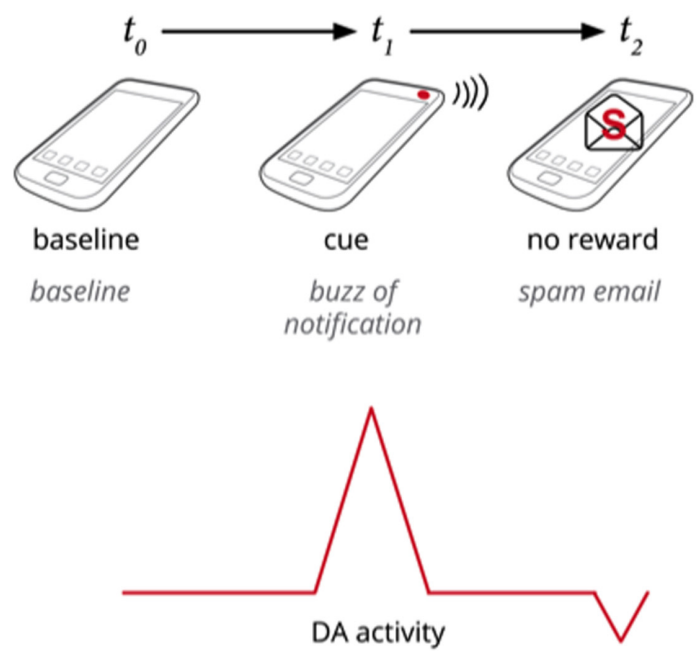

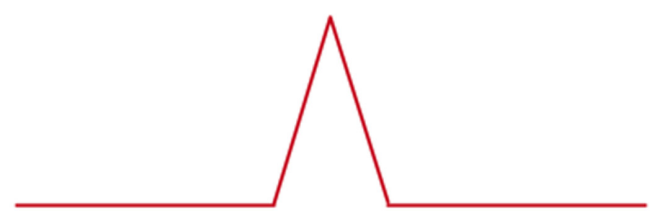

DA activity

\section{Negative Prediction Error Type 2}
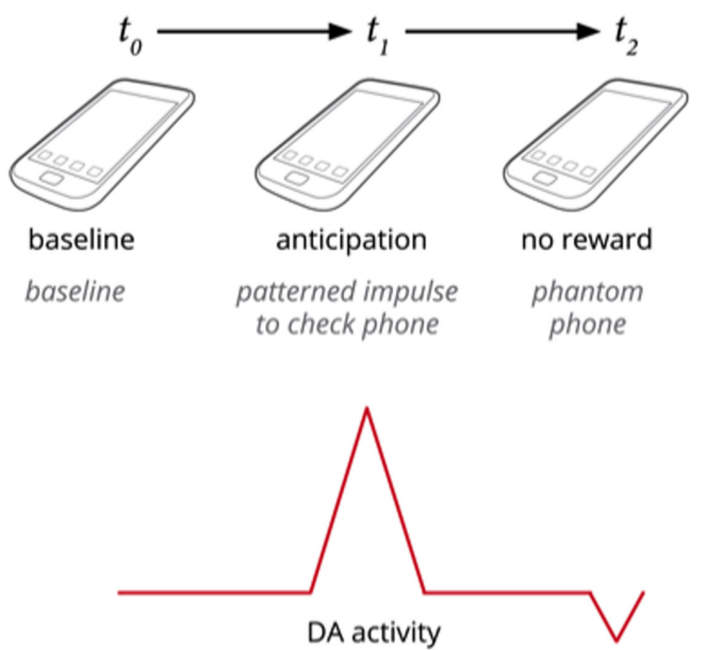

FIGURE 3 | (A-D) Presents an extrapolation of the data presented in Figure 2 to the present issue of smartphone addiction, whereby the dopamine activity increases at the anticipation of reward, and is reduced below baseline in cases where the expected reward is not met.

amidst current panics, we cannot dismiss the growing consensus described above on such negative outcomes as depression, anxiety, and loneliness.

Smartphone use and depression are strongly correlated, and one causal theory suggests that smartphones, which are frequently used to access social networks, provide a platform for which to frequently (often negatively) compare oneself to others (Steers et al., 2014). We have argued, however, that social monitoring is a fundamentally normal - indeed necessary - part of ordinary human cognition. Classical evolutionary accounts of this propensity have emphasized the human fondness for gossip (Dunbar, 2004) and social comparison (Festinger, 1954) as conferring adaptive advantages to assess threats, track trends and shifts in others' social status, and locate credible sources of cultural information and behavioral guides (Henrich, 2016). We add that comparing ourselves to others and against cultural norms also enables us to derive meaning, motivation, purpose, and a sense of identity. With socially connected smartphones, this evolutionary process simply runs on overdrive. We can now constantly and relentlessly engage in hyper-speed comparisons with social media content that is biased toward positivity. As media researchers have suggested, this continual stream of positive information about others allows users to repeatedly perform upward social comparisons and negative self-evaluations against a so-called "highlight reel" (Steers et al., 2014). Despite the obvious antigenic nature of cyber-mediated social comparisons, 
these accounts fail to acknowledge that the desire to socially connect is an even stronger motivator of smartphone use than the desire to do better than others.

To further address the non-benign concerns of smartphone overuse, the following section will once again employ theories of ordinary cognition to propose actions individuals can take to build happy, healthy relationships with mobile technology.

\section{FEEDING OUR HUNGRY GHOSTS}

If smartphone addiction rests on the fundamentally human proclivity toward prosociality, we can also learn to harness our social nature to pacify our cravings - or as Buddhic philosophies would put it, we can learn to sate our hungry ghosts.

In classical Buddhism, all creatures are said to undergo six life cycles, or go through six realms of existence (Levitt, 2003; Maté, 2008). They begin in Hell, where their life is described as constant torture, before moving on to the realm of Hungry Ghosts, where they are plagued by insatiable thirst, hunger, and cravings. Next comes the realm of Animals: a world of servitude and stupidity. This realm is followed by Asura, a world of anger, jealousy, and never-ending conflict. The Human realm comes next: a world of contradictions and indecisiveness; sweet and sour, hot and cold, happy and sad, good and evil. The human realm is a world of almost-thereness - wisdom and enlightenment are within reach, but never quite attained. Whether the next world of Deva-gati, or Heavenly Beings, offers final relief is open for debate (Levitt, 2003). It is world of intense pleasures, with intense miseries to match. Freedom from suffering, in the end, seems nowhere to be found. On a contemporary psychological reading, the Six Realms metaphor can also describe the quality and intentionality (aboutness) of the various states of consciousness and affect one will routinely encounter throughout the course of a day.

The Hungry Ghosts in this story can be understood as the state that regulates our cravings. This idea likely predates Buddhic philosophies, and is found in earlier Indian religions under the Sanskrit name Preta (Levitt, 2003). Pretas are supernatural creatures plagued by insatiable hunger and thirst. They have enormous stomachs, but very thin necks that can only support eating tiny things. In many Buddhist and Zen rituals, such as the Oryoki approach to eating and living, a single grain of rice is offered to Hungry Ghosts to acknowledge their existence and appease them a little (Levitt, 2003). The key here is to feed our Hungry Ghosts, and to find just the right amount. As we discuss further in our conclusion, this is consistent with harm-reduction approaches to addiction treatment that advocate responsible use over abstinence (Marlatt, 1996; Marlatt et al., 2011).

Recognizing smartphone cravings as Hungry Ghosts presents the opportunity to turn phone addiction into a intentional, justenough ritual.

\section{Set Intentional Protocols}

Many smartphone users feel trapped by their phones (Harmon and Mazmanian, 2013). The first step toward freedom from phone Hungry Ghosts, as we have seen, is to regain control of the pattern and make it predictable again. Switching off all sounds and notifications can help to 'un-ring' Pavlov's proverbial bell and cull habitual checking behaviors. As we described above, smartphone addiction is mediated by the grasp of intermittent reinforcement schedules of social rewards. With this in mind, setting regular intervals to check one's phone can reduce the strong cravings that arise from chaotic patterns of reward anticipation. When it comes to instant phonemediated communication, we can also make our intentions and expectations transparent, and agree on protocols with others. Clear workplace communication policies, for example, those that prohibit evening and weekend emails, or setting clear expectation for time-windows in replying have been shown to be effective in reducing stress and increasing productivity (Mark et al., 2012). Similar 'policies' and clear expectations for when to text or not to text - what we call 'intentional protocols' - can be devised among friends, families and lovers.

\section{CONCLUSION}

Like all natural proclivities, social monitoring and rehearsal can turn into Hungry Ghosts. The parallel with natural hunger and eating bear relevance to our argument about mobile technology. Blaming the rice, utensils, or kitchenware for one's insatiable gluttony does not so much deflate the problem as miss the mark entirely. The root of addictions, as we have seen, is not in substances or rewards themselves, and much less in the technologies that deliver such rewards, but in the anticipation of rewards and in delivery schedules and rituals. The hard truth about cravings is that they are ultimately self-referential: cravings are about cravings first and foremost.

Smartphones and mobile technologies are not the root cause of modern distress. In post-industrial environments where foods are abundant and readily available, our cravings for fat and sugar sculpted by distant evolutionary pressures can easily go into insatiable overdrive and lead to obesity, diabetes, and rampant heart disease (Henrich, 2016; Harari, 2017). As we argued in this paper, the prosocial needs and rewards of a physically weak species that relied on collective parenting (Hrdy, 2009) and distributed knowledge (Tomasello, 2014; Henrich, 2016) to survive and carve a moral niche in a harsh world can similarly be hijacked to produce a manic theater of hyper-social monitoring. Smartphones may be equated to hyper-efficient kitchenware. Both technologies help optimize the processing and delivery of specific kinds of basic needs: food on the one hand, and social information on the other. The key to eating well and being good social beings lies in finding the quality and intensity of consumption rituals. As in the oriyoki 'just the right amount' hungry ghost feeding ritual, the recipe lies in setting appropriate intentions, quality of awareness, and pacing for the time, place, and amount of information, connection, and comparison one will consume. Turning off notifications, as we have seen, has been shown to help users regain control of when and why to check their devices intentionally (Alter, 2017). When used to judicious social ends, smartphone and social media use can yield many positive outcomes, from increased subjective well-being (Kim and Lee, 2011) to better romantic relationships (Steers et al., 2014). 
To conclude, we recognize that there is a controversy in addiction research between abstinence-based and harmreduction approaches (Marlatt, 1996; Marlatt et al., 2011). The latter approach, which we advocate in this article, supports safe and responsible use, and consideration of the complexities of the social context in which people are drawn to substance use. While recent studies have shown that temporarily giving up certain social media activities could increase subjective wellbeing (see Alter, 2017, for a review), the professional and social consequences of giving up smartphone use altogether are currently not known, and are likely to be costly in a age that requires instant connection in so many domains of social life.

Individuals, rather, can mobilize their intrinsic drive toward sociality to mitigate the negative and increase the positive effects of smartphone use. Pursuing healthy social connection is the antidote. Rather than use smartphones to compare our lives to the distorted slice of reality others present, we can use them as communication tools to foster genuine emotional relationships. When competitive comparison seems inevitable, we can subvert into a motivator or reminder of our own unique skills - or better yet, we can cultivate genuine joy for the achievements of others (Chandra, 2017).

\section{AUTHOR CONTRIBUTIONS}

SV provided the theoretical framework based on his previous work on cultural affordances and internet sociality. MS

\section{REFERENCES}

Alter, A. (2017). Irresistible: The Rise of Addictive Technology and the Business of Keeping us Hooked. London: Penguin Books.

Andreassen, C. S., Billieux, J., Griffiths, M. D., Kuss, D. J., Demetrovics, Z., Mazzoni, E., et al. (2016). The relationship between addictive use of social media and video games and symptoms of psychiatric disorders: a large-scale crosssectional study. Psychol. Addict. Behav. 30, 252-262. doi: 10.1037/adb0000160 doi: $10.1037 / \mathrm{adb} 0000160$

Andreoni, J., and Vesterlund, L. (2001). Which is the fair sex? Gender differences in altruism. Q. J. Econ. 116, 293-312. doi: 10.1162/003355301556419

Andrews, P. W., Bharwani, A., Lee, K. R., Fox, M., and Thomson, J. A. Jr. (2015). Is serotonin an upper or a downer? The evolution of the serotonergic system and its role in depression and the antidepressant response. Neurosci. Biobehav. Rev. 51, 164-188. doi: 10.1016/j.neubiorev.2015.01.018

Andrews, P. W., and Thomson, J. A. Jr. (2009). The bright side of being blue: depression as an adaptation for analyzing complex problems. Psychol. Rev. 116, 620-654. doi: 10.1037/a0016242

Andrews, P. W., Thomson, J. A. Jr., Amstadter, A., and Neale, M. C. (2012). Primum non nocere: an evolutionary analysis of whether antidepressants do more harm than good. Front. Psychol. 3:117. doi: 10.3389/fpsyg.2012.00117

Atran, S., and Henrich, J. (2010). The evolution of religion: how cognitive byproducts, adaptive learning heuristics, ritual displays, and group competition generate deep commitments to prosocial religions. Biol. Theory 5, 18-30. doi: 10.1162/BIOT_a_00018

Belin, D., Jonkman, S., Dickinson, A., Robbins, T. W., and Everitt, B. J. (2009). Parallel and interactive learning processes within the basal ganglia: relevance for the understanding of addiction. Behav. Brain Res. 199, 89-102. doi: 10.1016/ j.bbr.2008.09.027

Bell, A. V., Richerson, P. J., and McElreath, R. (2009). Culture rather than genes provides greater scope for the evolution of large-scale human prosociality. Proc. Natl. Acad. Sci. U.S.A. 106, 17671-17674. doi: 10.1073/pnas.090323 2106 helped refine the theoretical framework and further ground it in neuroscience. SV and MS contributed equally to the writing.

\section{FUNDING}

This work was supported by Social Sciences and Humanities Research Council of Canada (MS) and the Healthy Brains for Healthy Lives Inititiative (SV).

\section{ACKNOWLEDGMENTS}

The authors wish to thank reviewers Giulia Piredda and Yasmina Jraissati and Associate Editor Maurizio Tirassa for their insightful comments and help in refining the argument presented here. We are much indebted to Maxwell Ramstead for his contribution to free-energy perspectives in our early work on Internetmediated sociality and for pointing us in the direction of the predictive-processing literature on addiction. SV wishes to express gratitude to Danny Frank for inviting him to present an early iteration of the social rehearsal theory of smartphone addiction at the Psychotherapy Rounds of the Jewish General Hospital in Montreal. Both authors are immensely grateful for the continued support and mentorship offered by Laurence Kirmayer at the Division of Social and Transcultural Psychiatry at McGill.

Billieux, J., Maurage, P., Lopez-Fernandez, O., Kuss, D. J., and Griffiths, M. D. (2015). Can disordered mobile phone use be considered a behavioral addiction? An update on current evidence and a comprehensive model for future research. Curr. Addict. Rep. 2, 156-162. doi: 10.1007/s40429-0150054-y

Boyer, P. (2008). Religion Explained. New York, NY: Random House.

Chandra, R. (2017). Facebuddha: Transcendence in the Age of Social Networks. San Francisco, CA: Pacific Heart Books.

dscout (2016). "Mobile Touches: dscout's Inaugural Study on Humans and Their Tech," Research Report. Available at: https://blog.dscout.com/hubfs/downloads/ dscout_mobile_touches_study_2016.pdf

Dunbar, R. I. (2004). Gossip in evolutionary perspective. Rev. Gen. Psychol. 8, 100-110. doi: 10.1037/1089-2680.8.2.100

Eckel, C. C., and Grossman, P. J. (1998). Are women less selfish than men?: Evidence from dictator experiments. Econ. J. 108, 726-735. doi: 10.1111/14680297.00311

Elhai, J. D., Dvorak, R. D., Levine, J. C., and Hall, B. J. (2017). Problematic smartphone use: a conceptual overview and systematic review of relations with anxiety and depression psychopathology. J. Affect. Disord. 207, 251-259. doi: 10.1016/j.jad.2016.08.030

Emanuel, R., Bell, R., Cotton, C., Craig, J., Drummond, D., Gibson, S., et al. (2015). The truth about smartphone addiction. Coll. Stud. J. 49, 291-299.

Espinosa, M. P., and Kováŕík, J. (2015). Prosocial behavior and gender. Front. Behav. Neurosci. 9:88. doi: 10.3389/fnbeh.2015.00088

Festinger, L. (1954). A theory of social comparison processes. Hum. Relat. 7, 117-140. doi: 10.1177/001872675400700202

Fiorillo, C. D., Tobler, P. N., and Schultz, W. (2003). Discrete coding of reward probability and uncertainty by dopamine neurons. Science 299, 1898-1902. doi: 10.1126/science. 1077349

Friston, K., and Kiebel, S. (2009). Predictive coding under the free-energy principle. Philos. Trans. R. Soc. Lond. B Biol. Sci. 364, 1211-1221. doi: 10.1098/rstb.2008. 0300 
Frith, C. (2002). Attention to action and awareness of other minds. Conscious. Cogn. 11, 481-487. doi: 10.1016/S1053-8100(02) 00022-3

Ginott, H. G. (1965/2009). Between Parent and Child: Revised and Updated: The Bestselling Classic That Revolutionized Parent-Child Communication. New York, NY: Harmony.

Harari, Y. N. (2017). Homo deus. Paris: Albin Michel.

Harmon, E., and Mazmanian, M. (2013). "Stories of the smartphone in everyday discourse: conflict, tension \& instability," in Proceedings of the SIGCHI Conference on Human Factors in Computing Systems, (New York, NY: ACM), 1051-1060.

Hebb, D. O. (1976). Physiological learning theory. J. Abnorm. Child Psychol. 4, 309-314. doi: 10.1007/BF00922529

Henrich, J. (2016). The Secret of Our Success: How Culture is Driving Human Evolution, Domesticating our Species, and Making us Smarter. Princeton, NJ: Princeton University Press.

Hrdy, S. B. (2007). "Evolutionary context of human development: the cooperative breeding model," in Family Relationships: An Evolutionary Perspective, eds C. A. Salmon and T. K. Shackelford (New York, NY: Oxford University Press), 39-68.

Hrdy, S. B. (2009). Mothers and Others. Cambridge, MA: Harvard UP.

Hussain, Z., Griffiths, M. D., and Sheffield, D. (2017). An investigation into problematic smartphone use: the role of narcissism, anxiety, and personality factors. J. Behav. Addict. 6, 378-386. doi: 10.1556/2006.6.201 7.052

Kawa, L. (2018). Two Major Apple Shareholders Push for Study of iPhone Addiction in Children. Available at: https://www.bloomberg.com/news/articles/2018-0108/jana-calpers-push-apple-to-study-iphone-addiction-in-children

Keiflin, R., and Janak, P. H. (2015). Dopamine prediction errors in reward learning and addiction: from theory to neural circuitry. Neuron 88, 247-263. doi: 10.1016/j.neuron.2015.08.037

Killingsworth, M. A., and Gilbert, D. T. (2010). A wandering mind is an unhappy mind. Science 330:932. doi: 10.1126/science. 1192439

Kim, J., and Lee, J. R. (2011). The facebook paths to happiness: effects of the number of facebook friends and self-presentation. Cyberpsychol. Behav. Soc. Netw. 14, 359-364. doi: 10.1089/cyber.2010.0374

Krach, S., Paulus, F. M., Bodden, M., and Kircher, T. (2010). The rewarding nature of social interactions. Front. Behav. Neurosci. 4:22. doi: 10.3389/fnbeh.2010. 00022

Laasch, O., and Conaway, R. (2009). Gender differences in preferences. J. Econ. Lit. 47, 448-474. doi: 10.1257/jel.47.2.448

Lee, Y. K., Chang, C. T., Lin, Y., and Cheng, Z. H. (2014). The dark side of smartphone usage: psychological traits, compulsive behavior and technostress. Comput. Hum. Behav. 31, 373-383. doi: 10.1016/j.chb.2013.1 0.047

Levitt, P. (2003). Fingerpainting on the Moon: Writing and Creativity as a Path to Freedom. New York, NY: Harmony.

$\mathrm{Li}$, S., and Chung, T. (2006). Internet function and internet addictive behavior. Comput. Hum. Behav. 22, 1067-1071. doi: 10.1016/j.chb.2004.0 3.030

Linnet, J. (2014). Neurobiological underpinnings of reward anticipation and outcome evaluation in gambling disorder. Front. Behav. Neurosci. 8:100. doi: 10.3389/fnbeh.2014.00100

Lopez-Fernandez, O., Honrubia-Serrano, L., Freixa-Blanxart, M., and Gibson, W. (2014). Prevalence of problematic mobile phone use in British adolescents. CyberPsychol. Behav. Soc. Netw. 17, 91-98. doi: 10.1089/cyber.2012. 0260

Lu, J. M., and Lo, Y. C. (2017). "Investigation of smartphone use while walking and its influences on one's behavior among pedestrians in Taiwan," in Proceedings of the International Conference on Human-Computer Interaction, (Cham: Springer), 469-475. doi: 10.1007/978-3-319-58753-0_67

Mar, R. A., Mason, M. F., and Litvack, A. (2012). How daydreaming relates to life satisfaction, loneliness, and social support: the importance of gender and daydream content. Conscious. Cogn. 21, 401-407. doi: 10.1016/j.concog.2011. 08.001

Mark, G., Voida, S., and Cardello, A. (2012). "A pace not dictated by electrons: an empirical study of work without email," in Proceedings of the SIGCHI Conference on Human Factors in Computing Systems, (Austin, TX: ACM), 555-564. doi: $10.1145 / 2207676.2207754$
Marlatt, G. A. (1996). Harm reduction: come as you are. Addict. Behav. 21, 779-788. doi: 10.1016/0306-4603(96)00042-1

Marlatt, G. A., Larimer, M. E., and Witkiewitz, K. (eds). (2011). Harm Reduction: Pragmatic Strategies for Managing High-Risk Behaviors. New York, NY: Guilford Press.

Maté, G. (2008). In the Realm of Hungry Ghosts. Berkeley, CA: North Atlantic Books.

Meier, S. (2007). Do women behave less or more prosocially than men? evidence from two field experiments. Public Finance Rev. 35, 215-232. doi: 10.1177/ 1091142106291488

Mercier, H., and Sperber, D. (2017). The Enigma of Reason. Cambridge: Harvard University Press.

Moll, H., and Tomasello, M. (2007). Cooperation and human cognition: the Vygotskian intelligence hypothesis. Philos. Trans. R. Soc. Lond. B Biol. Sci. 362, 639-648. doi: 10.1098/rstb.2006.2000

Mrazek, M. D., Phillips, D. T., Franklin, M. S., Broadway, J. M., and Schooler, J. W. (2013). Young and restless: validation of the Mind-Wandering Questionnaire (MWQ) reveals disruptive impact of mind-wandering for youth. Front. Psychol. 4:560. doi: 10.3389/fpsyg.2013.00560

Norenzayan, A., Henrich, J., and Slingerland, E. (2013). "Religious prosociality: a synthesis," in Cultural Evolution: Society, Technology, Language and Religion, eds P. J. Richerson and M. H. Christiansen (Cambridge, MA: MIT Press), 365-378. doi: 10.7551/mitpress/9780262019750.003. 0019

Norenzayan, A., and Shariff, A. F. (2008). The origin and evolution of religious prosociality. Science 322, 58-62. doi: 10.1126/science. 1158757

Oulasvirta, A., Rattenbury, T., Ma, L., and Raita, E. (2012). Habits make smartphone use more pervasive. Pers. Ubiquitous Comput. 16, 105-114. doi: 10.1007/s00779-011-0412-2

Pearson, C., and Hussain, Z. (2015). Smartphone use, addiction, narcissism, and personality: a mixed methods investigation. Int. J. Cyber Behav. Psychol. Learn. 5:17 doi: 10.4018/ijcbpl.2015010102

Poerio, G. L., and Smallwood, J. (2016). Daydreaming to navigate the social world: What we know, what we don't know, and why it matters. Soc. Pers. Psychol. Compass 10, 605-618. doi: 10.1111/spc3.1 2288

Ramstead, M. J., Veissière, S. P., and Kirmayer, L. J. (2016). Cultural affordances: scaffolding local worlds through shared intentionality and regimes of attention. Front. Psychol. 7:1090. doi: 10.3389/fpsyg.2016.01090

Ramstead, M. J. D., Badcock, P. B., and Friston, K. J. (2017). Answering Schrödinger's question: a free-energy formulation. Phys. Life Rev. doi: 10.1016/ j.plrev.2017.09.001 [Epub ahead of print].

Rand, D. G., Brescoll, V. L., Everett, J. A., Capraro, V., and Barcelo, H. (2016). Social heuristics and social roles: intuition favors altruism for women but not for men. J. Exp. Psychol. Gen. 145, 389-396. doi: 10.1037/xge000 0154

Richerson, P., Baldini, R., Bell, A. V., Demps, K., Frost, K., Hillis, V., et al. (2016). Cultural group selection follows Darwin's classic syllogism for the operation of selection. Behav. Brain Sci. 39:e58. doi: 10.1017/S0140525X1500 0606

Roberts, J. A., Pullig, C., and Manolis, C. (2015). I need my smartphone: a hierarchical model of personality and cell-phone addiction. Pers. Individ. Dif. 79, 13-19. doi: 10.1016/j.paid.2015.01.049

Sauer, V. J., Eimler, S. C., Maafi, S., Pietrek, M., and Krämer, N. C. (2015). The phantom in my pocket: determinants of phantom phone sensations. Mob. Media Commun. 3, 293-316. doi: 10.1177/2050157914562656

Seger, C. A. (2006). The basal ganglia in human learning. Neuroscientist 12, 285-290. doi: 10.1177/1073858405285632

Snodgrass, J. G., Lacy, M. G., Dengah, H. J., Batchelder, G., Eisenhower, S., and Thompson, R. S. (2016). Culture and the jitters: guild affiliation and online gaming eustress/distress. Ethos 44, 50-78. doi: 10.1111/etho.1 2108

Song, X., and Wang, X. (2012). Mind wandering in Chinese daily lives-an experience sampling study. PLoS One 7:e44423. doi: 10.1371/journal.pone. 0044423

Soutschek, A., Burke, C. J., Beharelle, A. R., Schreiber, R., Weber, S. C., Karipidis, I. I., et al. (2017). The dopaminergic reward system underpins gender 
differences in social preferences. Nat. Hum. Behav. 1, 819-827. doi: 10.1038/ s41562-017-0226-y

Steers, M. L. N., Wickham, R. E., and Acitelli, L. K. (2014). Seeing everyone else's highlight reels: how Facebook usage is linked to depressive symptoms. J. Soc. Clin. Psychol. 33, 701-731. doi: 10.1521/jscp.2014.33.8.701

The Economist (2017). Parents Now Spend Twice as Much Time with Their Children as 50 years ago. Available at: https://www.economist.com/blogs/graphicdetail/ 2017/11/daily-chart-20 [accessed January 22, 2018].

Thompson, S. H., and Lougheed, E. (2012). Frazzled by Facebook? an exploratory study of gender differences in social network communication among undergraduate men and women. Coll. Stud. J. 46, 88-98.

Tobler, P. N., O'Doherty, J. P., Dolan, R. J., and Schultz, W. (2006). Human neural learning depends on reward prediction errors in the blocking paradigm. J. Neurophysiol. 95, 301-310. doi: 10.1152/jn.00762.2005

Tomasello, M. (2009). Why We Cooperate. Cambridge, MA: MIT press.

Tomasello, M. (2014). A Natural History of Human Thinking. Cambridge, MA: Harvard University Press. doi: 10.4159/9780674726369

Tomasello, M., Melis, A. P., Tennie, C., Wyman, E., Herrmann, E., Gilby, I. C., et al. (2012). Two key steps in the evolution of human cooperation: the interdependence hypothesis. Curr. Anthropol. 53, 687-688. doi: 10.1086/ 668207

Tufekci, Z. (2008). Grooming, gossip, Facebook, and MySpace. Inf. Commun. Soc. 11, 544-564. doi: 10.1080/13691180801999050

Twenge, J. M. (2017). IGen: Why Today's Super-Connected Kids Are Growing Up Less Rebellious, More Tolerant, Less Happy-and Completely Unprepared for Adulthood-and What That Means for the Rest of Us. New York, NY: Simon and Schuster.

Van Deursen, A. J., Bolle, C. L., Hegner, S. M., and Kommers, P. A. (2015). Modeling habitual and addictive smartphone behavior: The role of smartphone usage types, emotional intelligence, social stress, self-regulation, age, and gender. Comput. Hum. Behav. 45, 411-420. doi: 10.1016/j.chb.2014. 12.039

van Holst, R. J., Veltman, D. J., Büchel, C., van den Brink, W., and Goudriaan, A. E. (2012). Distorted expectancy coding in problem gambling: is the addictive in the anticipation? Biol. Psychiatry 71, 741-748. doi: 10.1016/j.biopsych.2011. 12.030
Veissière, S. (2016a). "Varieties of tulpa experiences: the hypnotic nature of human sociality, personhood, and interphenomenality," in Hypnosis and Meditation: Towards an Integrative Science of Conscious Planes, eds A. Raz and M. Lifshitz (Oxford: Oxford University Press).

Veissière, S. (2016b). "The internet is not a river: space, movement and personhood in a wired world," in Click and Kin: Transnational Identity and Quick Media, eds M. Friedman and S. Schultermandl (Toronto: University of Toronto Press).

Veissière, S. P. L. (2017). Cultural Markov Blankets? Mind the Other Minds Gap!: comment on "Answering Schrödinger's question: a free-energy formulation" by Maxwell James Désormeau Ramstead et al. Phys. Life Rev. doi: 10.1016/j.plrev. 2017.11.001 [Epub ahead of print].

Weiser, E. B. (2015). \# Me: Narcissism and its facets as predictors of selfieposting frequency. Pers. Individ. Dif. 86, 477-481. doi: 10.1016/j.paid.2015. 07.007

West, G. L., Drisdelle, B. L., Konishi, K., Jackson, J., Jolicoeur, P., and Bohbot, V. D. (2015). Habitual action video game playing is associated with caudate nucleus-dependent navigational strategies. Proc. Biol. Sci. 282:20142952. doi: $10.1098 / \mathrm{rspb} .2014 .2952$

Whitehouse, H. (2004). Modes of Religiosity: A Cognitive Theory of Religious Transmission. Walnut Creek, CA: Rowman Altamira.

Yin, H. H., and Knowlton, B. J. (2006). The role of the basal ganglia in habit formation. Nat. Rev. Neurosci. 7, 464-476. doi: 10.1038/nrn1919

Zuriff, G. E. (1970). A comparison of variable-ratio and variable-interval schedules of reinforcement. J. Exp. Anal. Behav. 13, 369-374. doi: 10.1901/jeab.1970. 13-369

Conflict of Interest Statement: The authors declare that the research was conducted in the absence of any commercial or financial relationships that could be construed as a potential conflict of interest.

Copyright (อ 2018 Veissière and Stendel. This is an open-access article distributed under the terms of the Creative Commons Attribution License (CC BY). The use, distribution or reproduction in other forums is permitted, provided the original author(s) and the copyright owner(s) are credited and that the original publication in this journal is cited, in accordance with accepted academic practice. No use, distribution or reproduction is permitted which does not comply with these terms. 